\title{
Distribution and immunoregulatory properties of antisecretory factor
}

\author{
Todd S Davidson and William F Hickey \\ Department of Pathology, Borwell Building, Dartmouth Medical School —DHMC, Lebanon, NH 03756, USA
}

\begin{abstract}
Inflammatory processes and the mechanisms by which they are initiated and controlled within the central nervous system (CNS) involve a vast array of cell types and molecules. One cell type believed to be involved in the control of inflammation in the CNS is the microglial cell. The TLD antibodies are a panel of monoclonal antibodies reactive to rat microglial antigens. One antibody from this panel, clone TLD-1A8A, is specific for antisecretory factor (ASF). ASF is a previously identified protein characterized as a potent inhibitor of enterotoxin-induced intestinal fluid secretion. Our results extend the function of this molecule to include the regulation of immune reactions. Administration of TLD-1A8A to T-cell proliferation or mixed leukocyte response assays resulted in increased proliferation of T cells. Flow cytometric analysis indicates that ASF is expressed on macrophages, B cells and dendritic cells, but not on T cells or granulocytes. Immunohistochemical analysis indicates that ASF is expressed by macrophages and cells of dendritic morphology in the spleen, thymus, lymph nodes, Peyer's patch, and in the perivascular area in the CNS. Furthermore, Western blot analysis indicates that ASF is expressed in many tissues including all secondary lymphoid organs. These data suggest that ASF may have a previously unsuspected role in regulating the immune system.
\end{abstract}

Laboratory Investigation (2004) 84, 307-319, advance online publication, 26 January 2004; doi:10.1038/labinvest.3700036

Keywords: antisecretory factor; S5a; Rpn10; macrophage; immunosuppression; T-lymphocyte; cytokine

One of the major enigmas concerning inflammatory processes centers on the mechanisms whereby they are controlled and eventually shut down. A number of cytokines such as TGF- $\beta$ and IL-10 are known to have anti-inflammatory effects; ${ }^{1-3}$ however, there may exist other such molecules that are either undiscovered or whose anti-inflammatory properties are unknown. One molecule that has been characterized, but previously not known to have any influence on cells of the immune system, is antisecretory factor (ASF). ${ }^{4}$

ASF was identified by Lönnroth and Lange $^{5}$ as a protein produced by the pituitary and secreted into the blood stream possibly to counteract intestinal fluid secretion. The name originally assigned to the molecule derives from its potent effect in the system in which it was tested. ${ }^{5}$ In ligated loops of rat intestine, ASF acts as a potent inhibitor of mucosal fluid secretion induced by a number of enterotoxins including cholera toxin, okadaic acid or Clostridium difficile toxin A. ${ }^{4}$ No function in the immune system has been suggested for ASF.

Correspondence: Dr William Hickey, Department of Pathology DHMC, Lebanon, NH 03756, USA.

E-mail: william.f.hickey@dartmouth.edu

Received 27 October 2003; accepted 05 November 2003; published online 26 January 2004
In the central nervous system (CNS), microglial cells and perivascular cells have the potential to be important in inflammatory or reparative processes. $^{6,7}$ Immunological reactions in the CNS commonly involve perivascular and microglial cells as key participants. Among other activities, these cells are able to present antigen and elaborate cytokines, chemokines and reactive oxygen metabolites. ${ }^{8}$ To more completely understand the roles of CNS-derived microglia and perivascular cells, this laboratory had previously produced a panel of monoclonal antibodies (mAb) against cultured rat microglial cells. ${ }^{9}$ From this panel, a number of antibodies have been determined to bind with defined antigens, ${ }^{10}$ while others bind to undefined, functional molecules. ${ }^{11,12}$ In this latter group, there remain a significant number that bind to molecules not readily defined by immunohistochemical staining pattern or flow cytometric analysis. ${ }^{9}$ Some had unusual in vitro properties prompting additional studies in order to define their target antigen.

This report shows that TLD-1A8A specifically recognizes ASF. Further investigations with TLD1A8A show that ASF is expressed by macrophages, $B$ cells and dendritic cells, and modulates the proliferation of $\mathrm{T}$ cells. These data indicate that ASF has a heretofore unappreciated immunomodulatory function. 


\section{Materials and methods}

\section{Cell Culture}

The microglia cells used in this study were derived according to procedures described in detail elsewhere, ${ }^{9}$ which is a modification of the method of Giulian et al. ${ }^{13}$ Peritoneal exudate cells (PECs) were obtained by peritoneal lavage with ice-cold PBS of animals that had been challenged 5 days previously by ip injection of $3 \mathrm{ml}$ of aged 10\% Brewer's Thioglycolate (Difco, Detriot, MI, USA). NR8383 cells were obtained from the American Type Culture Collection (Manassas, VA, USA) and maintained in Hamm's F12 medium supplemented with 15\% FBS and 5\% NCTC-109, $5 \times 10^{-5} \mathrm{M}$ 2-mercaptoethanol, $2 \mathrm{mM}$ glutamine, $100 \mu \mathrm{g} / \mathrm{ml}$ penicillin, $100 \mu \mathrm{g} / \mathrm{ml}$ streptomycin and $100 \mu \mathrm{g} / \mathrm{ml}$ amphotericin B.

\section{Antibodies}

TLD-1A8A was derived from an immunization of mice with cultured rat microglial cells. ${ }^{9}$ To insure monoclonality of the cell line, TLD-1A8A was subcloned by limiting dilution and screened for secretion and specificity by immunohistochemistry. All experiments were performed with mAb derived from subcloned cells. Cells were grown in RPMI1640 supplemented with $20 \%$ FBS, $150 \mu \mathrm{g} / \mathrm{ml}$ oxalacetic acid, $5.5 \mu \mathrm{g} / \mathrm{ml}$ sodium pyruvate, $0.2 \mathrm{IU} / \mathrm{ml}$ insulin, $0.27 \mu \mathrm{g} / \mathrm{ml}$ hypoxanthine, $0.08 \mu \mathrm{g} / \mathrm{ml}$ thymidine, 5\% NCTC-109, $2 \mathrm{mM}$ glutamine, $100 \mu \mathrm{g} / \mathrm{ml}$ penicillin, $100 \mu \mathrm{g} / \mathrm{ml}$ streptomycin and $100 \mu \mathrm{g} / \mathrm{ml}$ amphotericin B. Antibody for immunohistochemistry, flow cytometry, Western blot and library screening was in the form of exhausted culture supernatant that had been filtered and contained $0.05 \%$ sodium azide as preservative. Culture supernatants were used straight for immunohistochemistry, diluted 1:2 for flow cytometry, and 1:5 for western blot and library screening. Ascites fluid was produced in pristane (Sigma) primed Balb/c mice. IgG was fractionated from the ascites fluid by precipitation with ammonium sulfate. IgG-containing fractions were collected and dialyzed against PBS. The antibody was subsequently purified from the resulting fraction by Protein $G$ affinity chromatography (Sigma), dialyzed against PBS, quantitated using the BCA method (Pierce) and adjusted to a concentration of $2 \mathrm{mg} / \mathrm{ml}$ in $\mathrm{PBS}$ and stored in aliquots at $-20^{\circ} \mathrm{C}$. Samples were verified to contain no endotoxin by the limulus amoebocyte lysate clot assay (Sigma). The isotype of TLD-1A8A is IgG1 as determined by direct ELISA using anti-isotype antibodies obtained from Southern Biotechnologies. MOPC-31 was obtained from Sigma for use as an isotype control and handled according to the supplier's instructions. OX-42 was used as exhausted supernatant and was prepared as above. RLN.9D3 and 8A2 were obtained from Serotec.

\section{cDNA Library Construction and Cloning of ASF}

Total and poly-A RNA were obtained from microglia using the guanidium-isothiocyanate and oligo(dt) methods (Five Prime Three Prime, Boulder, CO, USA) according to the manufacturer's instructions. A Lambda-ZAP cDNA library was kindly constructed in the laboratory of Doctor Srinivasa $R$ Nagalla using reagents and protocols from Stratagene. Escherichia coli strain $\mathrm{MRF}^{\prime}$ were infected with approximately $20000 \mathrm{pfu}$, plated on $150 \mathrm{~mm}$ Petri dishes in soft agar, and allowed to grow at $37^{\circ} \mathrm{C}$ until plaques were visible. Nitrocellulose membranes (Osmonics, Inc.) were impregnated with $10 \mathrm{mM}$ IPTG (Boehringer Mannheim, Germany) and laid onto the cultures to induce expression of the fusion protein while allowing adherence of the fusion protein to the membrane. After several hours the membranes were carefully lifted off, washed extensively with TBS containing 0.05\% Tween-20 (TTBS), blocked with TBS containing 1\% nonfat dry milk (blocking solution), and probed with TLD1A8A (in the form of culture supernatant) diluted 1:5 in blocking solution. The membranes were then washed extensively with TTBS and probed with goat anti-mouse peroxidase (Jackson ImmunoResearch, West Grove, PA, USA) diluted 1:5000 in blocking solution. After washing with TTBS, the membranes were developed with TMB substrate (KPL, Gaithersburg, MD, USA). Positive plaques were isolated and rescreened in the previous manner for several rounds at increasing dilutions until single plaques could be unambiguously identified and isolated. The positive plaques were then screened with the secondary antibody alone to confirm specificity for the primary. The plasmid pBK-CMV containing the insert was excised from the phage using protocols and reagents from Stratagene, and then purified from $E$. coli (Qiagen, Germany). The insert was then sequenced from both ends from the T3 and T7 primer sites with the ABI BigDye Terminator kit (Applied Biosystems, Foster City, CA, USA). The identity of the insert was determined using the BLAST search engine. $^{14}$

\section{Fusion Protein Generation and Ligated Loop Assay}

ASF was excised from pBK-CMV by codigestion with SalI and NotI and purified by gel electrophoresis. The insert was then subcloned into pGEX-5b (Amersham Biosciences). Sequencing using the start primer 5'-GGGCTGGCAAGCCACGTTTGGTG-3' was performed to verify that the insert was in-frame. The vector was then transfected into bacteria, grown in liquid culture and expression of the fusion protein was induced by addition of IPTG. Bacteria were collected by centrifugation after $4 \mathrm{~h}$ and lysed by sonication. The lysate was loaded onto a glutathione affinity column (Sigma), washed extensively, and eluted with excess glutathione. The eluate was 
dialyzed against PBS, quantitated and purity verified by gel electrophoresis. The ligated loop analysis was performed as described by Johansson et al. ${ }^{15}$ Briefly, animals were food-deprived overnight and anesthetized with nembutol. Loops of intestine, approximately $10 \mathrm{~cm}$ in length, were ligated with sutures and injected with $3 \mu \mathrm{g}$ okadaic acid, $0.3 \mu \mathrm{g}$ ASF-GST or $5 \mu \mathrm{g}$ purified TLD-1A8A as appropriate. After $90 \mathrm{~min}$, the animals were sacrificed and the loops excised, weighed, measured and photographed.

\section{T-cell Proliferation Assays}

Short-term antigen-specific T-cell lines were generated as previously described. ${ }^{16}$ The encephalitogenic peptide EP of guinea pig myelin basic protein GP68-88 (Y G S L P Q K S Q R S Q D E N P V V $\mathrm{H})^{17,18}$ was dissolved in PBS and emulsified with an equal amount of complete Freund's adjuvent supplemented with $5 \mathrm{mg} / \mathrm{ml}$ Mycobacterium tuberculosis strain H37RA at a final concentration of $1 \mathrm{mg} / \mathrm{ml}$ EP. The emulsion was injected intradermally into the footpad and at the base of the tail of female Lewis rats at $200 \mu \mathrm{g}$ EP per animal. On day 9, the animals were killed via anesthesia overdose and the draining popliteal and inguinal lymph nodes were aseptically removed. Single cells were isolated from the lymph nodes by mechanical disruption and placed in culture with $50 \mu \mathrm{g} / \mathrm{ml}$ EP in RPMI-1640 medium supplemented with $1 \%$ rat serum, $5 \%$ NCTC-109, $5 \times 10^{-5} \mathrm{M}$ 2-mercaptoethanol, $2 \mathrm{mM}$ glutamine, $100 \mu \mathrm{g} / \mathrm{ml}$ penicillin, $100 \mu \mathrm{g} / \mathrm{ml}$ streptomycin and $100 \mu \mathrm{g} / \mathrm{ml}$ amphotericin B (T-cell initiation/proliferation medium). On the third day of culture, dead cells were removed using Histopaque 1.077 (Sigma Chemical, St Louis, MO, USA) and antigen-specific blasts were recovered, washed with PBS and returned to culture without additional antigen in similar medium with the exception of 10\% FBS (HyClone Laboratories, Logan, UT, USA) replacing the $1 \%$ rat serum as well as supplementation of $5 \%$ exhausted medium from ConA stimulated splenocytes (T-cell expansion medium). The cells were split as necessary, and allowed to return to resting phase. Antigen-specific T cells were then recovered by centrifugation, washed with PBS and plated in sextuplet at $9 \times 10^{4} \mathrm{~T}$ cells/well in $250 \mu \mathrm{l}$ initiation medium, along with $6 \times 10^{5}$ irradiated (1500 rad) lymph node cells/well to serve as APC, in 96-well U-bottom plates (Falcon, Lincoln Park, NJ, USA). Antigen and antibodies were added at the indicated concentrations. At $48 \mathrm{~h}, 0.5 \mu \mathrm{Ci} /$ well ${ }^{3} \mathrm{H}$ thymidine (ICN Biomedicals, Inc., Costa Mesa, CA, USA, cat\#2403905) was added in $50 \mu$ l initiation medium. After $16 \mathrm{~h}$, the assay was harvested onto filters (LKB Wallac 1295-001 cell harvester) and radioactive incorporation was measured by liquid scintillation counting (Wallac 1205 Betaplate $^{\mathrm{TM}}$ ). Results are expressed as cpm.

\section{Mixed Lymphocyte Response Assay}

Lewis and DA(RP) rats share the RT1.B1 and RT1.D1 loci at MHC II, but differ at their MHC I loci. ${ }^{19}$ $\mathrm{DA}(\mathrm{RP})$ rats were sensitized to Lewis antigens by repeated intravenous and intraperitoneal injection of Lewis leukocytes. Spleens from the immunized DA(RP) animals (responder) and naïve Lewis animals (stimulator) were aseptically recovered. The Lewis spleen was irradiated (1500 rad). Single-cell suspensions were obtained by mechanical disruption. The cells were washed extensively and resuspended in T-cell proliferation medium. Responder cells were plated at the indicated concentrations along with $2 \times 10^{5}$ stimulator cells in $200 \mu \mathrm{l}$ in 96-well U-bottom plates (Falcon, Lincoln Park, NJ, USA). Antibodies were added as $50 \mu \mathrm{l}$ of exhausted culture supernatant. At $72 \mathrm{~h}, 0.5 \mu \mathrm{Ci} /$ well ${ }^{3} \mathrm{H}$-thymidine (ICN Biomedicals, Inc., Costa Mesa, CA, USA, cat\#2403905) was added in $50 \mu \mathrm{l}$. After $16 \mathrm{~h}$, the assay was harvested onto filters (LKB Wallac 1295-001 cell harvester) and radioactive incorporation was measured by liquid scintillation counting (Wallac 1205 Betaplate $^{\mathrm{TM}}$ ). Results are expressed as cpm.

\section{Flow Cytometry}

Cells for flow cytometric analysis were washed with ice-cold PBS, resuspended in 10\% FBS-PBS at $1 \times 10^{6}$ cells $/ \mathrm{ml}$, dispensed into Falcon 2059 tubes at $5 \times 10^{5}$ cells/test, and incubated on ice for 30-45 min. The cells were then centrifuged, resuspended in the appropriate antibody solution and incubated on ice for $30-45 \mathrm{~min}$. Antibodies were in the form of exhausted culture supernatants that were diluted with an equal volume of $10 \%$ FBSPBS. Controls consisted of no antibody, isotype control primary plus secondary antibody, and secondary antibody alone. The cells were then washed twice with PBS, resuspended in $200 \mu \mathrm{l}$ of a 1:200 dilution of goat anti-mouse FITC (Sigma) diluted in $10 \%$ FBS-PBS, and incubated on ice for 30-45 min. The cells were then washed three times with PBS, fixed in $2 \%$ paraformaldehyde, and stored in the dark at $4^{\circ} \mathrm{C}$ until analyzed. In the case of two-color flow cytometry, the same protocol was employed except that the antibodies were directly labeled using the Zenon ${ }^{\circledR}$ labeling kit according to the manufacturer's instructions (Molecular Probes). Cells were analyzed on a FACScan (Becton-Dickinson). A total of 5000 events/sample were collected.

\section{Confocal Microscopy}

Staining was performed as for flow cytometry, with the inclusion of $0.2 \%$. saponin to permeabilize the cells. After washing and fixing, $10 \mu \mathrm{l}$ of sample was pipeted onto a microscope slide and allowed to dry. 
Coverslips were mounted using antifade mounting medium (Molecular Probes) and allowed to set. Images were obtained on a Zeiss confocal microscope and analyzed using Adobe Photoshop.

\section{Immunohistochemistry}

Animals were killed by an anesthesia overdose. Organs were removed, embedded in Tissue-Tek OCT medium (Miles, Elkhart, IN, USA) and snap frozen. In all. 6- $\mu$ m-thick sections were cut, mounted onto glass slides, fixed briefly with methanol, and put into a blocking solution of $10 \%$ FBS in $0.5 \mathrm{M}$ Tris, $\mathrm{pH}$ 7.6. The tissue sections were then covered with the antibody solution and incubated overnight at $4^{\circ} \mathrm{C}$. The next day, the sections were washed with blocking solution, then overlaid with rat absorbed, biotinylated anti-mouse antibody (Vector Laboratories, Burlingame, CA, USA) at room temperature for $45 \mathrm{~min}$. Following the incubation, the sections were again washed with blocking solution and then placed into a solution of absolute methanol, 6\% $\mathrm{H}_{2} \mathrm{O}_{2}$ for $15 \mathrm{~min}$ to remove endogenous peroxidase activity. The sections were then washed with Tris buffer, followed by blocking solution, then overlaid with ABC reagent (Vector) for $1-1.5 \mathrm{~h}$ at room temperature Following washes in blocking solution, color was developed using 3,3'-diaminobenzidine in the presence of $\mathrm{H}_{2} \mathrm{O}_{2}$. The sections were then dehydrated through a series of ethanol solutions and xylene, cover slipped and then examined by light microscopy.

\section{Western Blot}

Freshly isolated organs were carefully weighed and dissolved in $1 \mathrm{ml}$ of Tris buffered saline (TBS) containing $2 \%$ Triton X-100, 1\% NP-40, $0.5 \%$. SDS, $0.5 \%$ Tween-20, $1 \mathrm{mM}$ DTT and a protease inhibitor cocktail (Roche, Indianapolis, IN, USA) per gram of tissue, homogenized, and remaining particulate matter removed via centrifugation. In total, $10 \mu \mathrm{m}$ of lysate, with the exception of spleen that was diluted four-fold relative to the other samples, was combined with reducing sample buffer, boiled for 3-5 min, separated by PAGE, and then blotted to nitrocellulose. Unoccupied binding sites were blocked with $5 \%$ nonfat dry milk in TTBS (blocking buffer), whereupon the blot was probed with a 1:5 dilution of exhausted TLD-1A8A supernatant diluted in blocking buffer. The blot was washed with TTBS, probed with donkey anti-mouse peroxidase (Jackson) diluted 1:5000 in blocking buffer, washed again, and bands were visualized by ECL and autoradiography.

\section{Results}

\section{TLD-1A8A Enhances T-cell Proliferation}

To identify immunoregulatory molecules, antibodies from the TLD series ${ }^{9}$ were screened for their ability to modulate the proliferation of antigen-specific CD4 + Th1 cells. Short-term T-cell lines were derived from immunized Lewis rats and expanded in vitro in the presence of the immunizing antigen. Antigen-driven proliferation was then assessed in the presence of the appropriate TLD antibody or isotype matched control antibody. As anticipated, several of the mAbs from this series blocked T-cell proliferation, probably by interfering with T-cellAPC interactions ${ }^{11}$ and data not shown. In contrast, clone TLD-1A8A had an opposite effect. TLD-1A8A, but not an isotype matched control antibody, enhanced the proliferation of $\mathrm{T}$ cells (Figure 1a). This effect was not due to endotoxin contamination of the antibody sample as determined by Limulus amoebocyte lysate clot assay (data not shown). Furthermore, the ability of TLD-1A8A to enhance T-cell proliferation was not related to the specificity of the TCR as the antibody had the same effect on T cells specific for a variety of antigens (data not shown).

A similar augmenting effect of the antibody was noted in mixed leukocyte assays (Figure 1b). The assay used made use of the MHC II syngeneic, MHC I allogeneic Lewis and DA(RP) rat strains. ${ }^{19} \mathrm{DA}(\mathrm{RP})$ rats were sensitized with cells from Lewis animals to expand the repertoire of $\mathrm{T}$ cells reactive to Lewis antigens. Splenocytes from the immunized DA(RP) animals were then plated onto irradiated splenocytes from Lewis animals in the presence of TLD-1A8A or an isotype control antibody. Again, TLD-1A8A, but not an isotype control antibody, increased T-cell proliferation.

\section{TLD-1A8A Recognizes Antisecretory Factor}

TLD-1A8A was used to screen a cDNA Lambda-ZAP (Stratagene) bacterial expression library constructed with cDNA derived from cultured rat microglia. Two separate screenings yielded a total of seven phage clones. The plasmids contained within the phagemids were excised and the inserts sequenced from both ends. All clones were identical, and identified by BLAST analysis ${ }^{14}$ as encoding rat ASF. Compared to the sequence cloned here, the sequence of ASF published in Genbank (accession number AB017188) contains an additional 61 nucleotides of $5^{\prime}$ sequence, and an additional nine nucleotides of $3^{\prime}$ sequence, presumably due to the restriction enzymes used in the preparation of the microglial library. The only difference between the published ASF sequence and the isolated phagemid insert is a single $G$ to $A$ substitution in the $3^{\prime}$ noncoding region. The nucleotide sequence throughout the entirety of the predicted coding region is identical. BLAST analysis also revealed that the $26 \mathrm{~S}$ proteasome regulatory subunit S5a (Genbank accession number AF175575) shares a very high degree of homology with ASF. S5a and ASF can be distinguished by the absence of nine nucleotides in the $3^{\prime}$ 
a

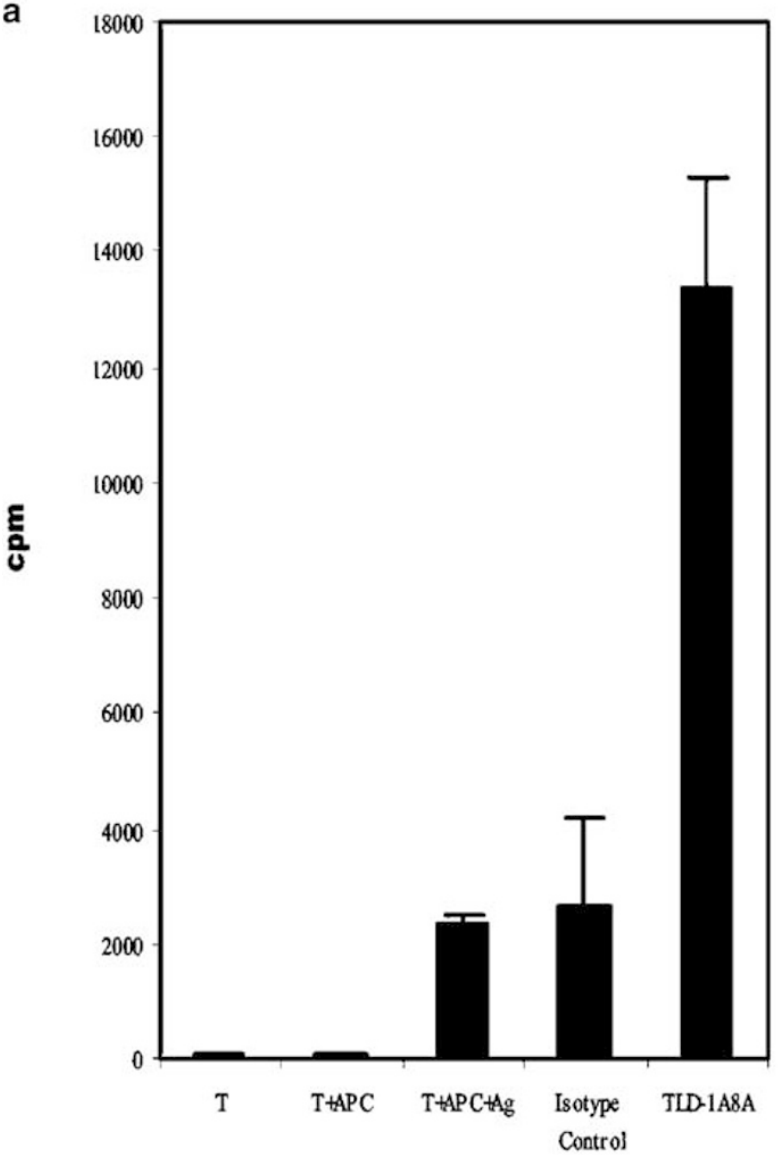

b

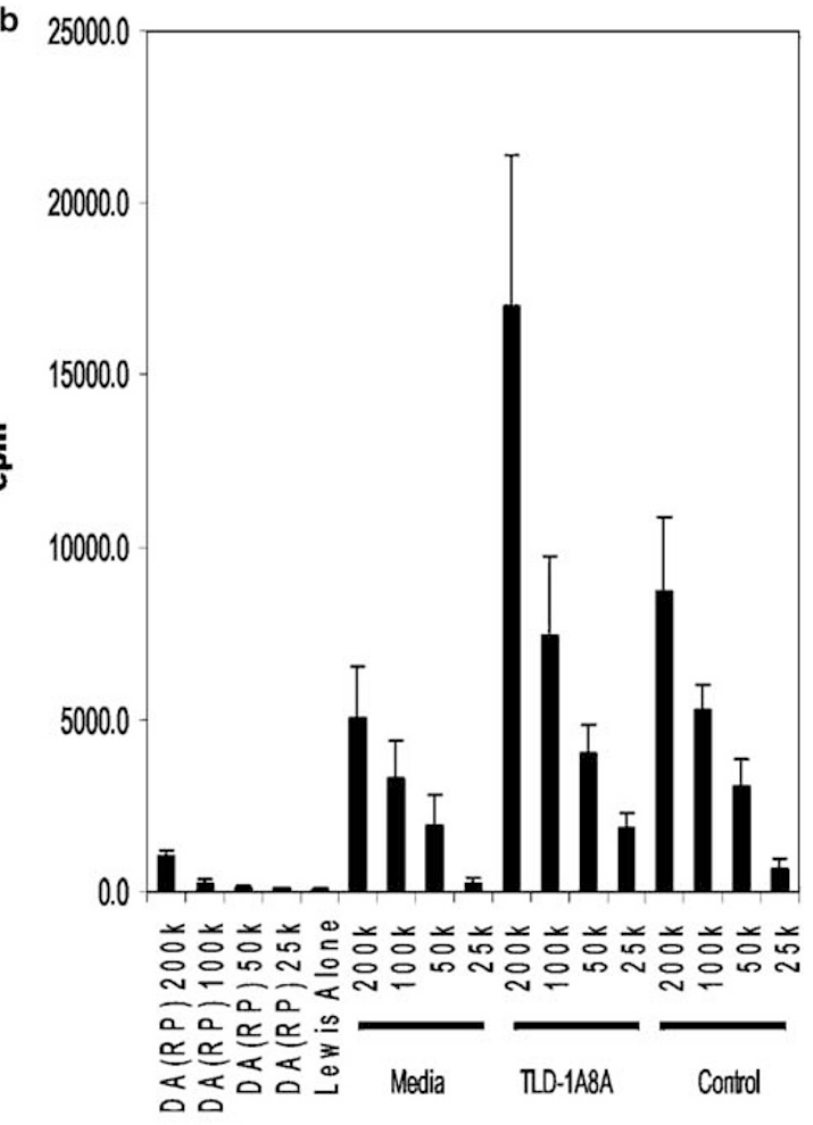

Figure 1 TLD-1A8A increases antigen-driven T-cell proliferation as well as MLR. (a) Short-term T-cell lines were derived from draining lymph nodes of rats immunized with antigen in CFA and expanded in vitro as described in Materials and methods. A total of $9 \times 10^{4} \mathrm{~T}$ cells were plated with $6 \times 10^{5}$ irradiated lymph node cells as APC at six wells per condition. Antigen was added at a concentration of $50 \mu \mathrm{g} / \mathrm{ml}$ and antibodies were included at a concentration of $20 \mu \mathrm{g} / \mathrm{ml}$. At $48 \mathrm{~h}$, the plate was pulsed with $0.5 \mu \mathrm{Ci} /$ well ${ }^{3} \mathrm{H}$-thymidine and harvested $16 \mathrm{~h}$ later onto filters. Incorporation was measured by scintillation counting. Results are expressed as cpm. Data are representative of six independent experiments. TLD-1A8A compared to isotype control $P=4 \times 10^{-7}$. (b) DA(RP) rats were sensitized with cells from Lewis rats in order to expand the pool of T cells responsive to Lewis antigens. In all, $7 \times 10^{4}$ spleen cells from the immunized animals were plated on varying numbers of irradiated Lewis spleen cells. Each condition was replicated in sextuplet. Antibodies were added at $20 \mu \mathrm{g} / \mathrm{ml}$. At $72 \mathrm{~h}$, the plate was pulsed with $0.5 \mu \mathrm{Ci} /$ well ${ }^{3} \mathrm{H}$-thymidine and harvested $16 \mathrm{~h}$ later onto filter paper. Incorporation was measured by scintillation counting. Results are expressed as cpm. $P=0.0006,0.002,0.0006$ and $6 \times 10^{-5}$ for $200 \mathrm{k}$, $100 \mathrm{k}, 50 \mathrm{k}$ and $25 \mathrm{k}$, respectively, for TLD-1A8A compared to isotype control.

coding portion of the S5a molecule, resulting in a deletion of three amino acids. As the sequence obtained here contains these nine nucleotides, we identify the antigen recognized by TLD-1A8A as ASF, and not S5a. Figure 2a compares the gene sequence derived from our screening of the Lewis rat microglial library to the ASF sequence derived from rat intestine cDNA library and the sequence of S5a.

In order to confirm that the gene that we obtained indeed codes for a protein possessing antisecretory function, and that our antibody reacts with this protein, we generated an ASF-GST fusion protein from the sequence derived from the microglial library. ASF was subcloned into pGEX-5 and transfected into bacteria for expression of the fusion protein. ASF-GST was purified by glutathione affinity chromatography and purity was verified by gel electrophoesis. The purified fusion protein was then used in the ligated loop assay as described by
Johansson et $a l^{20}$ using okadaic acid as the enterotoxic agent. ${ }^{20}$ As seen in Figures 2a and b, ligated loops of intestine that are injected only with PBS have a grossly normal appearance, displaying signs of hemorrhage only at the sites of ligation. However, loops that have been challenged with okadaic acid for 90 min exhibit signs of necrosis throughout the entirety of the loop (Figure 2b). ASF-GST is able to completely counter the effects of okadaic acid, resulting in a grossly normal appearing intestine (Figures $2 \mathrm{~b}$ and $\mathrm{c}$ ). However, injection of a combination of okadaic acid, ASF-GST and TLD-1A8A resulted in a necrotic loop of intestine (Figures $2 \mathrm{~b}$ and d), indicating that TLD-1A8A neutralized ASFGST and is able to block its functions in vivo.

The gross morphology results are mirrored by intestinal fluid secretion. The loops shown in Figure $2 \mathrm{~b}$ were weighed and measured and their mass per length was calculated. Loops that had been 
a

Coned wh TLO.1.. Antisecretory Factor $\$ 5 \mathrm{a}$

doned wit tio Antisecretony Factor $\$ 5 \mathrm{a}$

doned wht Tlo i. Antisecretory Facto

Goned with TiL i. Antisecretory Factor 53

doned whth TLD i... 53

Goned with Tilo i... Antisecretory Factor $\$ 53$

doned with Tito i.... Antisecretory Factor

oloned with tio 1 Antisecretony Factor

doned with Tlói. Antisecretony Factor "doned whth Tiloi...

doned whth TLD 1 ... Antisecretory Factor $55 \mathrm{a}$

doned wath Tíb $1 . .$. ny facto

doned with Tibi. Antisecretory Factor

doned wht tio i. Antisecretory Factor doned with TLibi... Antisecretory Factor

doned with Tiloi... Antisecretory Factor 5

doned with TLo i.... Antisecretory Factor $\$ 53$

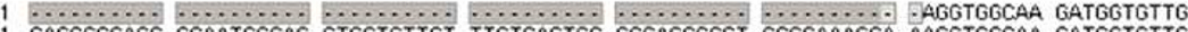
GAGCGCCAGG CCAATCGGAG GTGCTGTTGT TTGTCAGTCC CGGAGCCGGT CCGCAAAGGA AAGGTGGCAA GATGGTGTTG

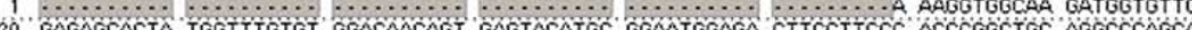
1. 1 GAGAGCACTA TGG 22 . 100 GGatGciolc aAcat 61 GGATOCTOTC AACATTOTAT OTCACTCAAA GACCCQAAGC AACCCTOAGA ATAACGTGG CTTGATCACG CTGGCCAATO O2 180 ACTGTGAGGT GCTGACCACA CTCACCCCTG ACACTGGCCG AATCCTCTCC AAGCTCCACA CTGTCCAGCC CAAAGGCAAD 241 ACTGTGAGGT GCTGACCACA CTCACCCCTG ACACTGGCCG AATCCTCTCC AAGCTCCACA CTGTCCAGCC CAAAGGCAAD 182 ACTGTGAGGT GCTGACCACA CTCACCCCTG ACACTGGCCG AATCCTCTCC AAGCTCCACA CTGTCCAGCC CAAAGGCAAG

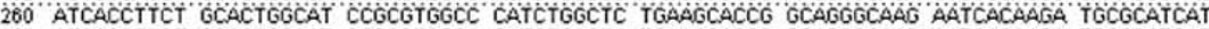
321 ATCACCTTCT GCACTGGCAT CCGCGTGGCC CATCTGGCTC TGAAGCACCG GCAGGGCAAG AATCACAAGA TGCGCATCAT 262 ATCACCTTCT GCACTGGCAT CCGCGTGGCC CATCTGGCTC TGAAGCACCG GCAGGGCAAG AATCACAAGA TGCGCATCAT 340 CGCCTTCGTG GGTAGCCCTG TGGAGGACAA CGAGAAGGAT CTGGTGAAAC TAGCTAAACG CCTTAAGAAA GAAAAAGTGA 401 CGCCTTCGTG GGTAGCCCTG TGGAGGACAA CGAGAAGGAT CTGGTGAAAC TAGCTAAACG CCTTAAGAAA GAAAAAGTGA 342 CGCCTTCGTG GGTAGCCCTG TGGAGGACAA CGAGAAGGAT CTGGTGAAAC TAGCTAAACG CCTTAAGAAA GAAAAAGTGA 420 ATGTTGACAT CATCAATTTT GGGGAAGAAGG AGGTGAACAC AGAGAAGCTG ACAGCCTTTO TOGACACATT AAACGGCAAG 41 AT 1 422 S0 GACGGAAC 561 GACGGAACTG GATCACATCT AJTGACAGTG CCTCCTGGGC CCAGCCTGGC CGATGCTCTC ATCAGTTCTC CTATTCTAGC . .

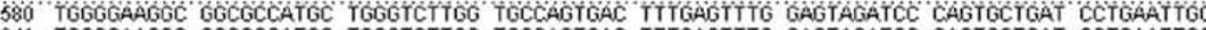
641 TGGGGAAGGC GGCGCCATGC TGGGTCTTGG TGCCAGTGAC TTTGAGTTTG GAGTAGATCC CAGTGCTGAT CCTGAATTGG 382 TGGGGAAGGC GGCGCCATGC TGGGTCTTGG TGCCAGTGAC TTTGAGTTTG GAGTAGATCC CAGTGCTGAT CCTGAATTGO

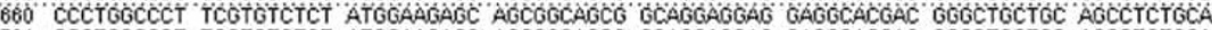
721 CCCTGGCCCT TCGTGTCTCT ATGGAAGAGC AGCGGCAGCG GCAGGAGGAG GAGGCACGAC GGGCTGCTGC AGCCTCTGCA 662 CCCTGGCCCT TCGTGTCTCT ATGGAAGAGC AGCGGCAGCG GCAGGAGGAG GAGGCACGAC GGGCTGCTOC AGCCTCTGCA 740 GCTGAGGCTG GAATTGCCAC ACCTGGGACT GAAGGTGAAC GAGACTCAGA TGATGCCCTA CTGAAGATGA CCATCAGCCA 301 GCTGAGGCTG GAATTGCCAC ACCTGGGACT GAAGGTGAAC GAGACTCAGA TGATGCCCTA CTGAAGATGA CCATCAGCCA 742 GCTGAGGCTG GAATTGCCAC ACCTGGGACT GAAC...... ... ACTCAGA TGATGCCCTA CTGAAGATGA CCATCAGCCA

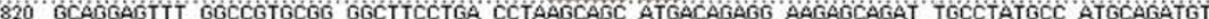
O1 GCAGGAGTTT GOCCOTGCGG GOCTTCCTTA CCTAAOCACC ATCACAOAGG AAOAOCAOAT TOCCTATOCD ATOCAGATOT ( 00 . OCOCTGCHOSGO 作 O 作 980 AAGGAGGAGG ATGACTATGA CGTGATGCAG GACCCAGAGT TCCTTCAGAU CGTCCTAGAG AACCTTCCCG GTGTGGATCC 1041 AAGGAGGAGG ATGACTATGA CGTGATGCAG GACCCAGAGT TCCTTCAGAG CGTCCTAGAG AACCTTCCCG GTGTGGATCC 73 AAGGAGGAGG ATGACTATGA CGTGATGCAG GACCCAGAGT TCCTTCAGAG CGTCCTAGAG AACCTTCCCG GTGTGGATCC

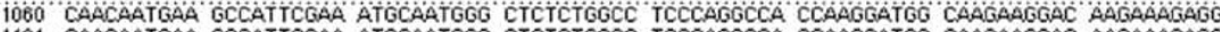
1121 CAACAATGAA GCCATTCGAA ATGCAATGGG CTCTCTGGCC TCCCAGGCCA CCAAGGATGG CAAGAAGGAC AAGAAAGAGG 1053 CAACAATGAA GCCATTCGAA ATGCAATGGG CTCTCTGGCC TCCCAGGCCA CCAAGGATGG CAAGAAGGAC AAGAAAGAGG 1140 AAGAGAAGAA GTGAGGCCGG AGGGAGGGGT AGCCGAGTCT GCCCTGGGGA CTGTAAGGAT GGGGTTGGAT AAAGACTTAG 1201 AAGAGAAGAA GTGAGGCCGG AGGGAGGGGT AGCCGABTCT GCCCTGGGGA CTGTAAGGAT GGGGTTGGAT AAAGACTTAG

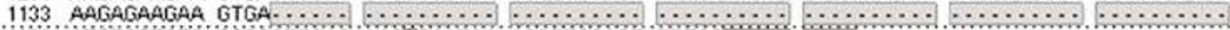

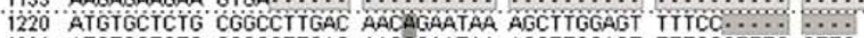
1281 ATGTGCTCTG CGGCCTTGAC AACGGAATAA AGCTTGGAGT TTTCCCTTTG CTTC b
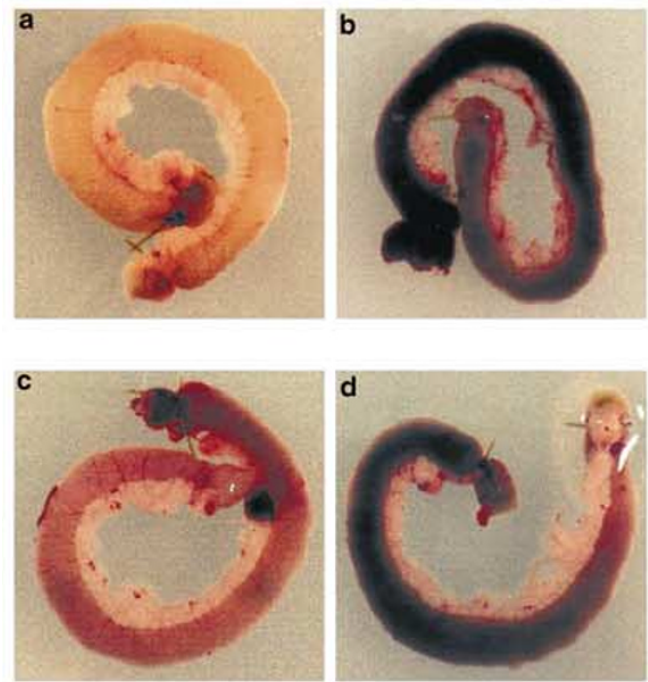

C

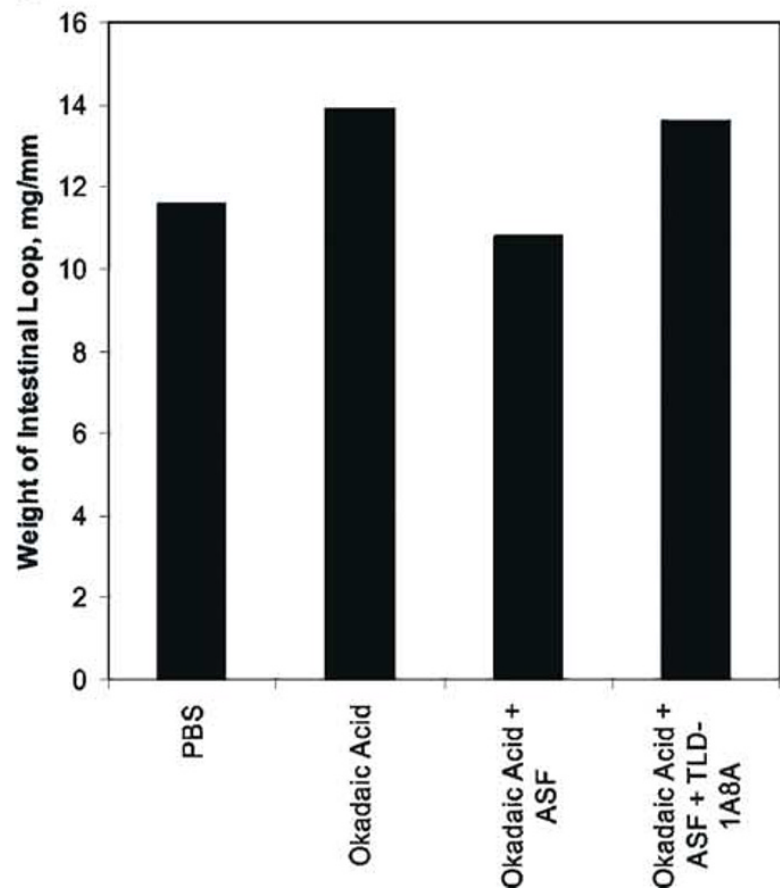


challenged with okadaic acid had greater mass per length than loops that had been challenged with PBS, indicating fluid secretion into the lumen. ASFGST was able to block this fluid secretion. The combination of okadaic acid, ASF-GST and TLD1A8A resulted in fluid secretion, indicating that TLD-1A8A blocked the effect of ASF (Figure 2c).

\section{In Situ Distribution of ASF}

In the spleen (Figure 3a), TLD-1A8A prominently stained a circle of large, stellate macrophages in the marginal sinus of the white pulp, as well as scattered macrophages in both the red and white pulp. In the thymus (Figure $3 \mathrm{~b}$ ), prominent cytoplasmic staining of large branching cells, abundant in the medullary areas, was observed. Scattered, elongate cells were also positive in the cortical zones. A similar staining pattern was seen in lymph nodes, where TLD-1A8A stained large cells of dendritic morphology in germinal centers, large cells in the subcasular sinus, and scattered macrophages in the T-cell zones and hilar area (Figure 3c). In the intestine, the antibody gave strong staining of a central population of large cells in Peyer's patches,
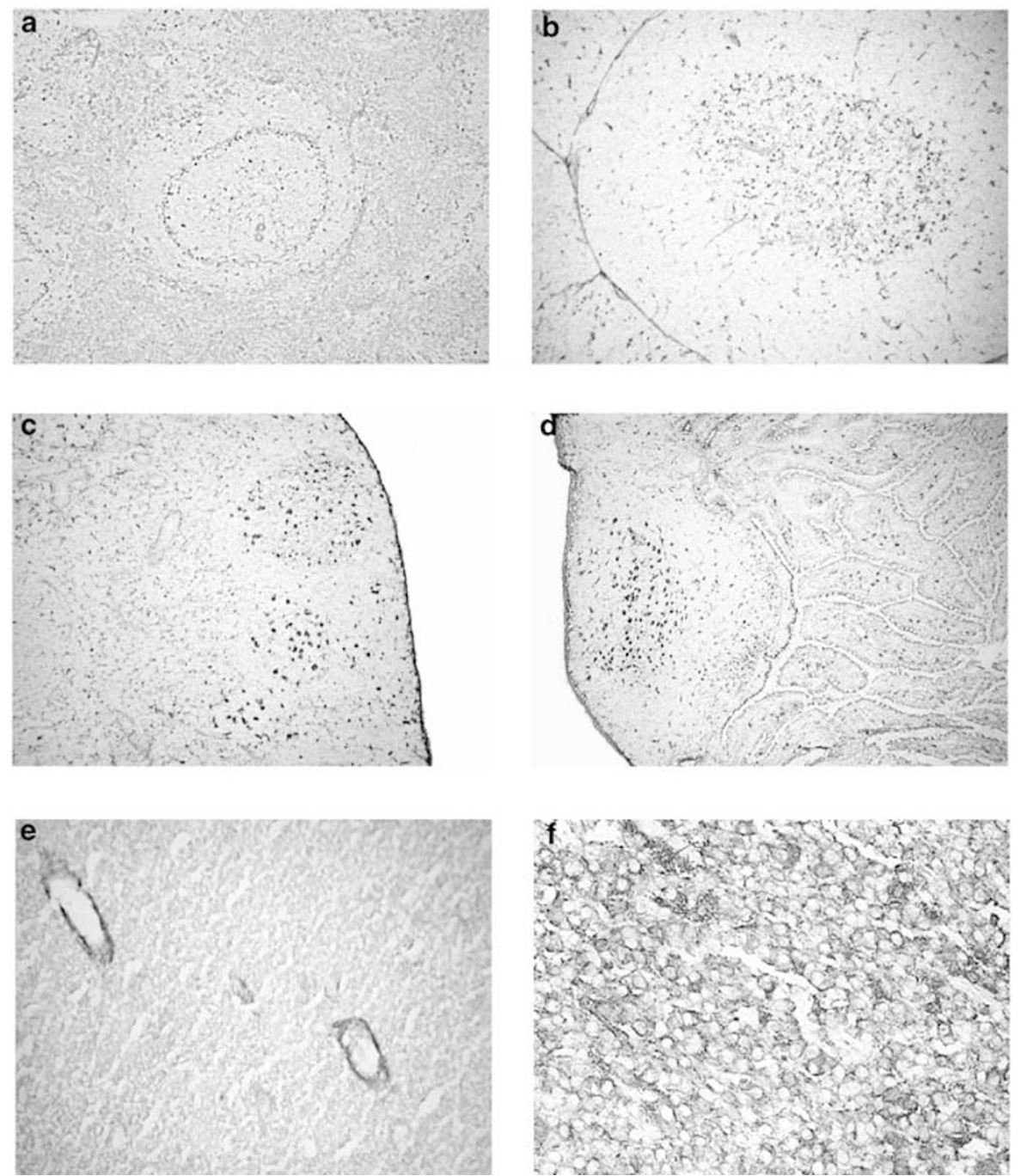

Figure 3 Immunohistochemical distribution of ASF. Tissues were processed for immunohistochemistry as described in Materials and methods. Photomicrographs were captured digitally and imported in Adobe Photoshop ${ }^{\mathrm{TM}}$ for analysis. Representative sections of each organ are depicted. (a) Spleen, $10 \times$ objective, (b) thymus, $10 \times$ objective, (c) lymph node, $10 \times$ objective, (d) intestine with Peyer's patch, $10 \times$ objective, (e) CNS, $40 \times$ objective, (f) pituitary, $40 \times$ objective.

Figure 2 TLD-1A8A recognizes ASF. (a) Comparison of the sequence derived from the rat microglial cDNA library as having reactivity to TLD-1A8A to the previously published sequence of ASF and S5a. Seven identical clones from two independent screenings of the cDNA library contained identical sequences. As indicated, the sequence cloned with TLD-1A8A is identical to ASF, with the exception of the missing $3^{\prime}$ and $5^{\prime}$ sequence. ASF and S5a can be distinguished by the deletion of nine nucleotides in the S5a molecule relative to ASF at position 776 of S5a. (b) Ligated loops of rat intestine that have been challenged for 90 min with PBS (a), okadaic acid (b), okadaic acid and ASF-GST (c) or okadaic acid, ASF-GST and TLD-1A8A (d). (c), Mass per length of the loops shown in (b). 


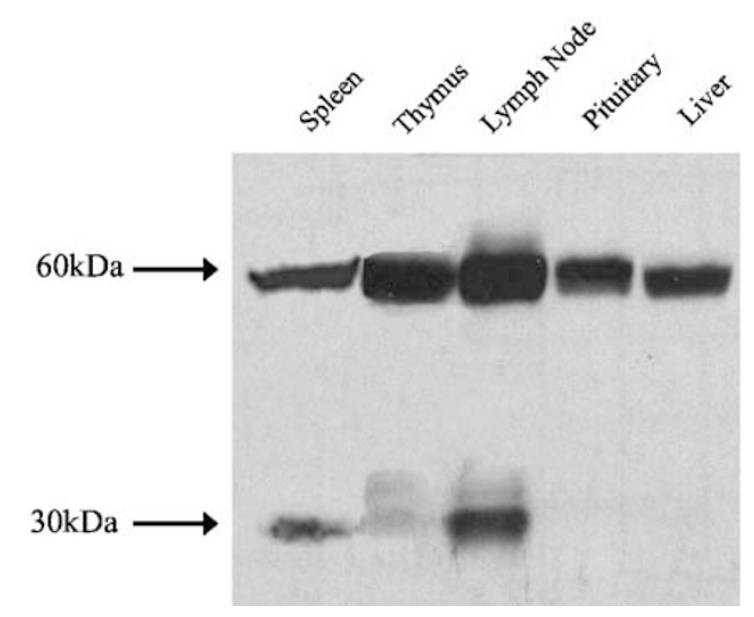

Figure 4 Western blot analysis of ASF expression. In all, $10 \mu \mathrm{g}$ of total lysate from the thymus, lymph node, pituitary and liver, and $2.5 \mu \mathrm{g}$ of total lysate from the spleen were analyzed by Western blot with TLD-1A8A. Spleen was analyzed independently of the other samples, and thus is juxtaposed accordingly. The higher molecular weight band is at approximately $60 \mathrm{kDa}$. The lower molecular weight band seen in the spleen, lymph node and, to a lesser extent, thymus is at $30 \mathrm{kDa}$.

and scattered macrophages in the connective tissue cores of the villi (Figure 3d). Moreover, the apex of the mucosal cells overlying Peyer's patches was also positive, while the species of mucosal cells on the villi stained less intensely or were negative. In the CNS, blood vessels and perivascular cells were TLD1A8A positive, while parenchymal microglia, astrocytes and neurons were negative (Figure 3e). Immunohistochemical staining of the pituitary resulted in a pattern of diffuse, granular cytoplasmic positivity of virtually all cells of the adenohypophysis (Figure 4f), as previously reported for ASF. ${ }^{4}$

\section{Western Blot Analysis of ASF Expression}

The expression of ASF was also determined in various tissues by Western blot analysis using lysates from the spleen, thymus, lymph node, pituitary and liver. TLD-1A8A detected a band of approximately $60 \mathrm{kDa}$ in all samples, with particularly strong expression in the spleen (Figure 4). The predicted molecular weight of ASF is $41 \mathrm{kDa}$, with the apparent molecular weight being higher due to glycosylation. ${ }^{4}$ A band of approximately $30 \mathrm{kDa}$ was also detected in the spleen, lymph node and, to a lesser extent, thymus, but not in the pituitary or the liver. It is undetermined whether the lower molecular weight band is a result of pre- or posttranslational processing, a degradation product, or a reflection of differentially regulated gene expression.

\section{Flow Cytometric Analysis of ASF Expression}

The immunohistochemical analysis strongly suggested that ASF is expressed by cells of the monocyte/macrophage lineage. Cells isolated by peritoneal lavage of rats challenged 5 days previously intraperitoneally with thioglycolate are predominantly activated macrophages and granulocytes that can be distinguished on the flow cytometer on the basis of their differential forward versus side scatter (Figure 5a). In these mixed populations, TLD-1A8A stained exclusively the macrophage population, but not the granulocyte population (Figure 5b). Both macrophages and granulocytes stained with MRC OX-42. ${ }^{21}$ The rat alveolar macrophage cell line NR8383 ${ }^{22}$ stained with TLD-1A8A (Figure 5c) while peripheral blood mononuclear cells did not (data not shown). Neither mitogen-activacted (Figure $5 \mathrm{~d}$ ) nor resting T-cells were stained by TLD-1A8A (Figure 5e), although all cells in both populations were positive when stained with the anti-rat TcR $\alpha / \beta$ antibody R7.3. Only a minimally detectable expression was noted on cultured microglial cells (Figure 5g). This is somewhat surprising as TLD-1A8A was generated by immunizing mice with rat microglia, and ASF was cloned from a rat microglial library. This observation may be explained by the activation state of the cell. Microglia produced under our culture conditions are not activated, and it may be that activation of the cell (such as might occur upon injection of the cell into a xenogeneic animal) upregulates the expression of the molecule.

Two-color flow cytometry was used to identify the cell types expressing ASF in the spleen and lymph node (Figure 6). In both the spleen and lymph node, subpopulations of $\mathrm{CD} 11 \mathrm{~b}$ positive macrophages are ASF positive (Figures 6a and b). There are also subpopulations of RLN.9D3 positive B cells in both organs that express ASF (Figures $6 \mathrm{c}$ and d). In contrast, the majority of CD11c positive dendritic cells express the ASF molecule (Figures 6e and f).

\section{Confocal Analysis of ASF Expression}

Thioglycolate elicited PECs, or NR8383 macrophages were permeabilized with $0.2 \%$ saponin, stained with TLD-1A8A followed by goat antimouse FITC and analyzed by confocal microscopy. Images obtained from these studies are shown in Figure 7. The majority of ASF is localized within the cell in a perinuclear distribution, although some cells clearly have ASF associated with the cell membrane. As shown already, flow cytometry demonstrates that there is indeed ASF associated with the cell surface. Depending on the particular cell being analyzed, the staining can either be punctate or diffuse.

\section{Discussion}

ASF is a previously identified protein whose function had heretofore only been associated with control of intestinal mucosal secretion in response 
a

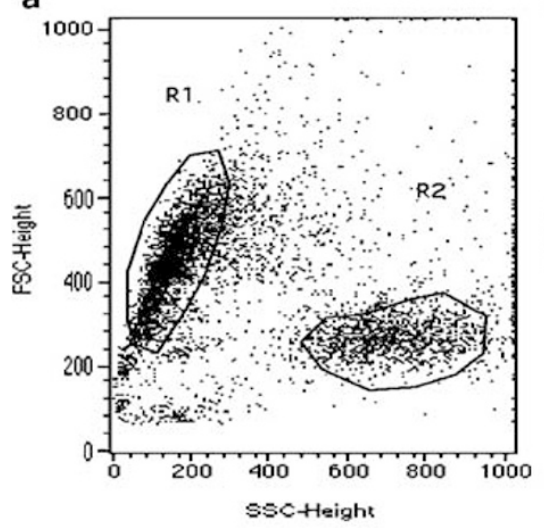

b

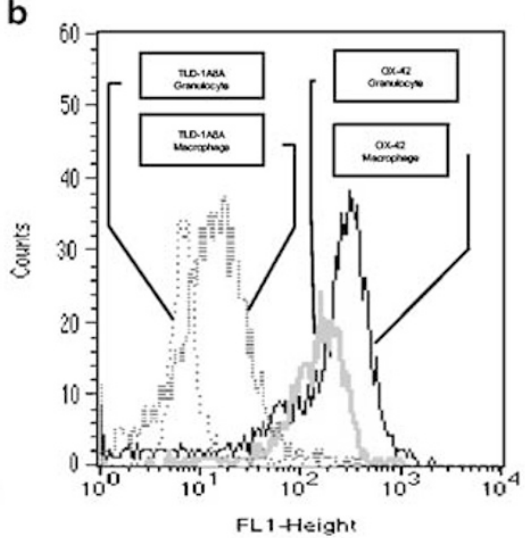

C

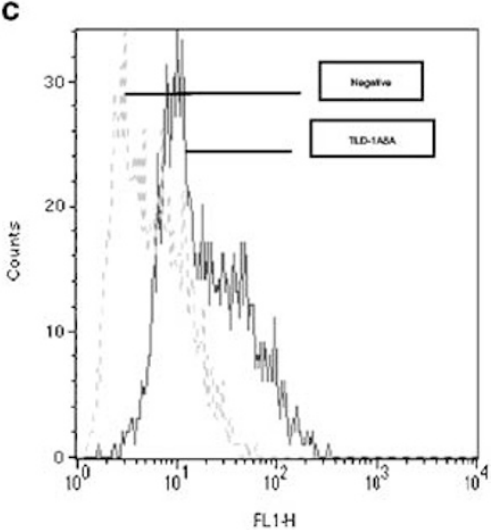

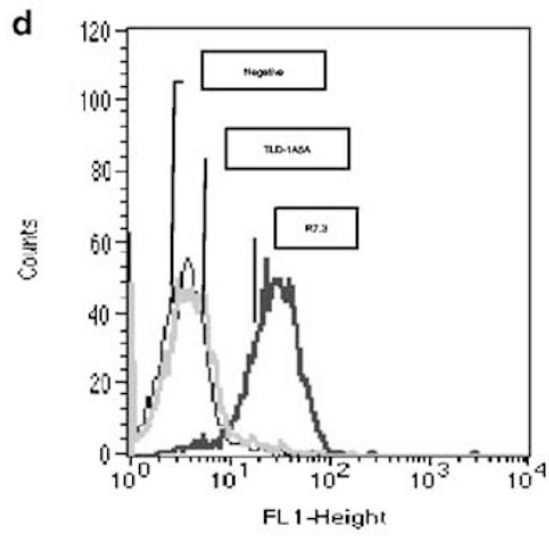

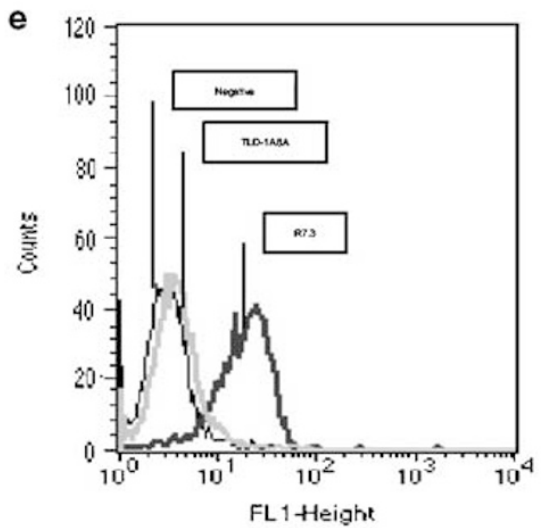

f

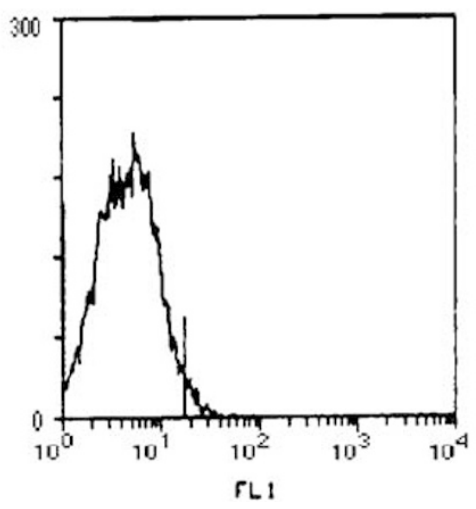

g

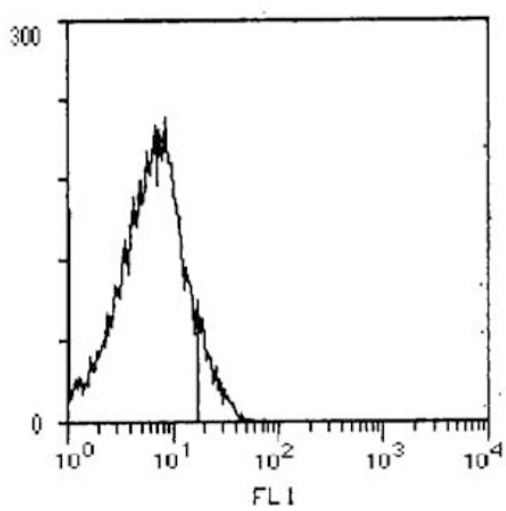

Figure 5 Flow cytometric analysis of ASF expression. (a) Forward vs side scatter plot of thioglycolate-induced PECs. Region R1 indicates the macrophages population and region R2 indicates the granulocyte population. (b) FL1 channel of the populations depicted in (a). (c) ASF expression on NR8383 macrophages. ASF is not expressed on activated (d), or resting (e) T cells. Negative control (f) and TLD-1A8A (g) on cultured rat microglia.

to enterotoxins. ${ }^{4}$ However, it also appears to have a role in modulating immunological reactions. ASF is expressed by cells of the immune system, predominantly members of the monocyte/macrophage family, and appears to regulate the proliferation of $\mathrm{T}$ cells. Two questions concerning this curious protein immediately arise: does it represent a single gene product or is it a member of a larger, novel family of proteins? And what function, or functions, does it serve in vivo?
cDNA sequences with homology to ASF have been identified in mammalian and non-mammalian eukaryotes, but not in prokaryotes, and have been suggested to have a regulatory role in the proteasome. These ASF-like sequences have been identified as S5a, Rpn10, Psmd-1, multiubiquitin chain binding protein (mcb-1), sun-1 and p50. These are identical at their $\mathrm{N}$-termini, but differ to varying degrees at their C-termini. Five Rpn10 transcripts have been described in the mouse, named Rpn10a- 
a

Spleen

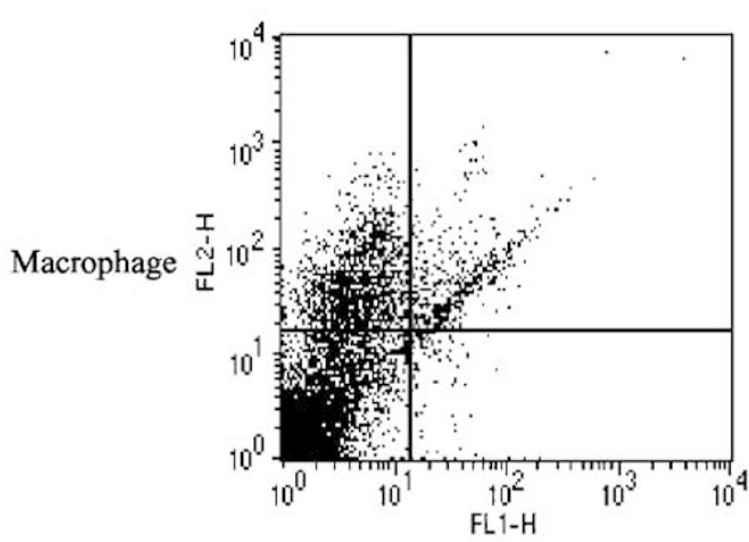

C

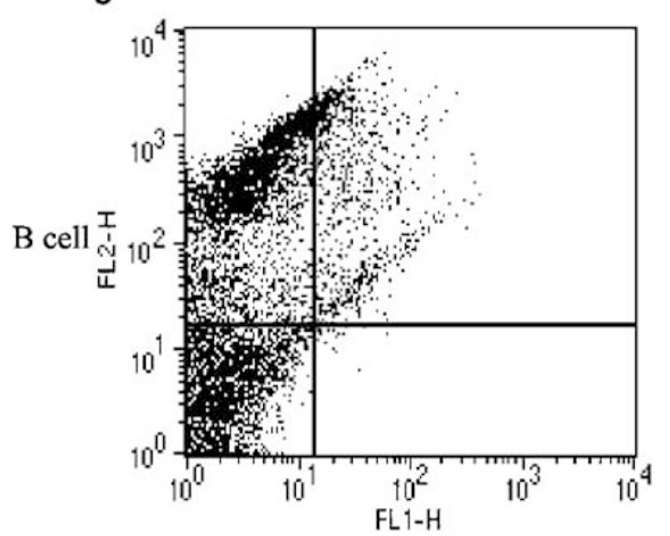

e

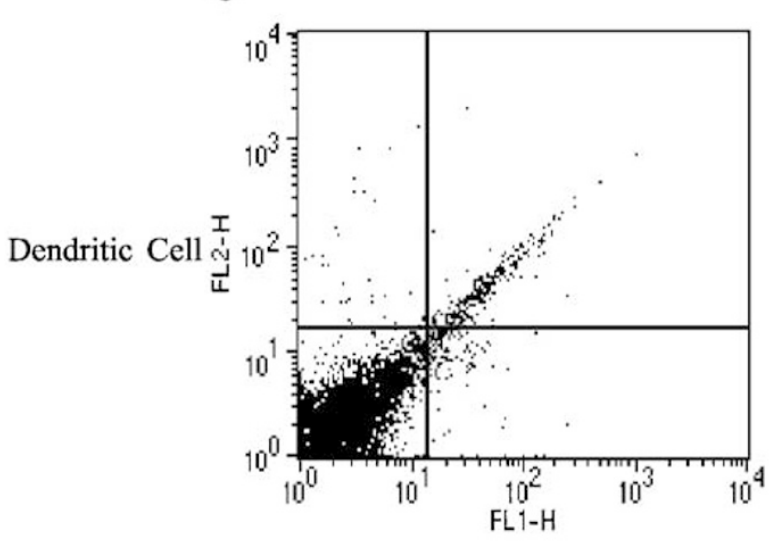

b Lymph Node

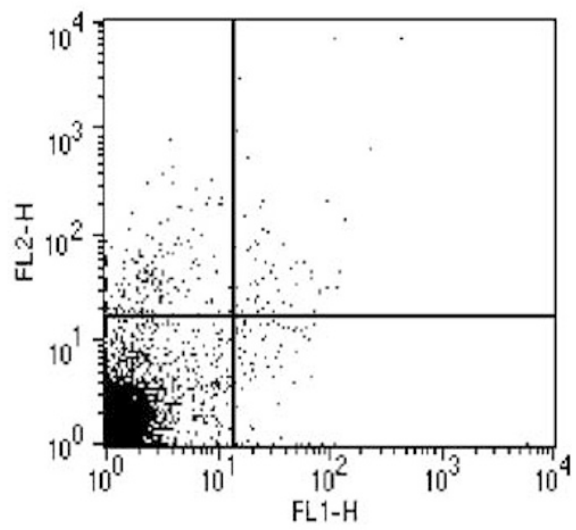

d

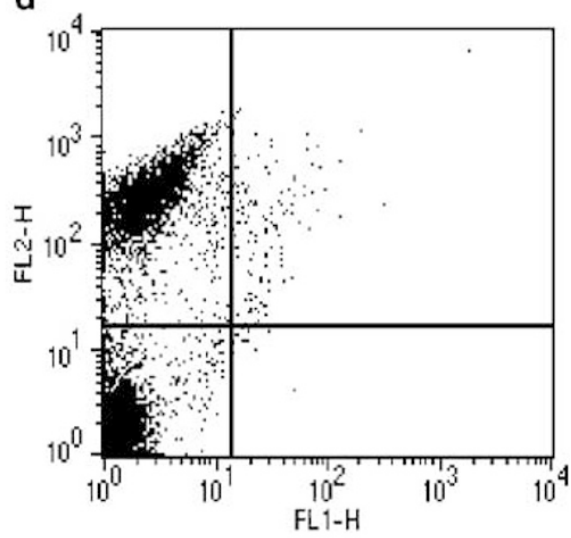

f

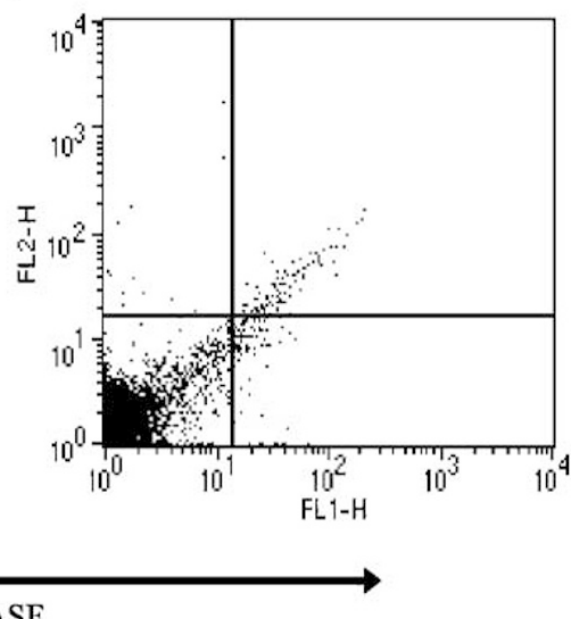

Figure 6 Two-color flow cytometry of ASF expression. Single-cell suspensions were prepared from spleens (left column) and lymph nodes (right column) and analyzed for ASF expression. Macrophages were identified with OX-42 (a) and (b). B cells were identified with RLN.9D3 (c) and (d). Dendritic cells were identified with 8A2 (e) and (f).

Rpn10e, and are generated by alternative splicing at the $3^{\prime}$ end of the transcript. ${ }^{23}$ Despite its proposed role in the regulation of the $26 \mathrm{~S}$ proteasome, deletion of the Rpn10 gene in yeast produces no detectable effect on growth or degradation of proteins in that system. ${ }^{24}$ In counterpoint, Kawahara et $a l^{23}$ observed embryonic lethality in mice made deficient in Rpn10. It should be noted that the cDNA 


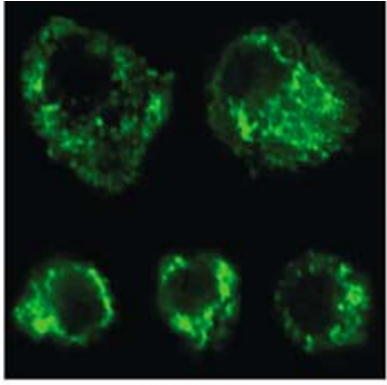

NR8383

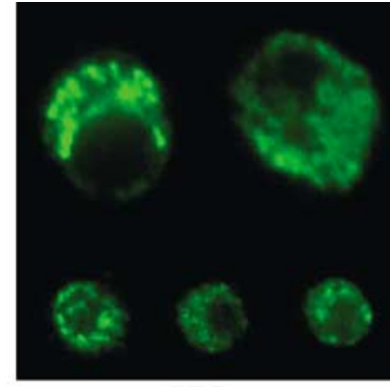

PEC
Figure 7 Confocal analysis of ASF expression. NR8383 macrophages (left) and PECs (right) were stained with TLD-1A8A as described in Materials and methods.

that we isolated is $100 \%$ identical to ASF in the coding region, and not to the other genes.

According to the analysis performed by Lange and Louriroth, ${ }^{4}$ several potential ASF genes (or ASF-like genes) are located in the human genome on chromosomes 1, 19 and 23 with pseudogenes located on chromosomes 10 and 15. A recent query of electronic databases finds ASF genes located on human chromosome 2q34. In concurrence with Lange et al, we find that there is a high probability that a von Willenbrand type A (vWA) domain is located in the ASF molecule at its N-terminus. As vWA domains mediate protein-protein interactions, this suggests that ASF might interact with other proteins via its $\mathrm{N}$-terminus. In addition, PROSITE ${ }^{25}$ analysis indicates the possibility of two PKC, 12 CK2, one amidation and eight myristylation sites in the ASF molecule. However, this must be interpreted with caution as all of these domains are very small and thus likely to occur by chance. There is no detectable leader sequence to direct the molecule to the cell surface or for secretion.

ASF appears to modulate the proliferation of $\mathrm{T}$ cells. In in vitro assays, TLD-1A8A had the effect of increasing T-cell proliferation. These assays made use of primed and differentiated $\mathrm{T}$ cells and thus measured the ability of ASF to control the proliferation of memory/effector cells. As these cells are critical in orchestrating immunological functions, future studies addressing the ability of ASF to control T-cell responses in vivo are warranted.

The proliferation assays presented here were performed in $1 \%$ rat serum. We have found there to be batch-to-batch variability with regard to the presence or absence of ASF in serum as detected by Western blot. Previous literature suggests that serum ASF is regulated by food intake. ${ }^{26-28}$ However, we found no difference in serum ASF levels between animals that have been food-deprived overnight versus $2 \mathrm{~h}$ postprandial animals (data not shown). Thus, the reason for this variability of serum ASF levels remains unresolved. When performing T-cell proliferation assays using serum in which ASF is present, TLD-1A8A did not increase proliferation (data not shown). TLD-1A8A only enhanced proliferation when using serum in which ASF was not detected. TLD-1A8A also consistently enhanced proliferation when the assay was performed using the serum-free medium AIM-V. This held true for the MLR results as well. Thus, naturally occurring serum ASF appears able to inhibit TLD-1A8A competitively.

The immunohistochemical distribution of ASF suggests a role for this molecule in the immune system. In lymphoid organs, such as the spleen, thymus and lymph nodes, ASF expression is prominent in macrophages and in cells with dendritic morphology. In the CNS, perivascular cells, critical for the initiation and regulation of inflammatory reactions, ${ }^{8}$ express ASF. The Peyer's patch in the intestine, important for the immunological surveillance of the intestinal environment, appears to be a prominent site of ASF expression. If the epitope recognized by TLD-1A8A is not conserved in alternatively spliced forms of ASF, or if not all members of an 'ASF family' (if such exists) of proteins carry the epitope, then its level of expression or tissue distribution may be difficult to define using TLD-1A8A alone.

It should be noted that the flow cytometry experiments were performed on viable, nonpermeabilized cells and would only detect ASF associated with the cell surface. We cannot determine the exact nature of this association with these data alone, be it transmembrane or some other form of linkage. Sequence analysis did not identify a transmembrane domain, suggesting either a GPI linkage or some other sort of nontransmembrane association with the cell surface. However, the fact that ASF is associated with the cell surface suggests the molecule would be available to participate in intercellular molecular interactions.

The intracellular localization of ASF could also be of importance. The intracellular distribution of the molecule suggests that it is localized within the Golgi apparatus, although this has not been confirmed. It is also unclear whether this indicates a role for ASF in Golgi function, or if the assay was merely detecting ASF being newly synthesized.

Although ASF was detected on the cell surface of macrophages as well as intracellularly, it may also act as a soluble molecule. Indeed, soluble ASF has been demonstrated in plasma, ${ }^{28}$ and has antisecretory activity in the rat intestinal loop experimental model. TLD-1A8A did not stain T cells, indicating that the in vitro effect of enhancing T-cell proliferation was not due to a direct binding of the mAb to the $\mathrm{T}$ cell itself. Rather, this result is more probably due to TLD-1A8A binding to the APC-associated and/or soluble ASF, thereby exerting an indirect effect on the $\mathrm{T}$ cell. Three hypothetical mechanisms might account for this observation. First, there may be a receptor for the ASF molecule on the T cell, and disrupting this receptor-ligand interaction with TLD-1A8A results in blockade of a normally 
suppressive signal. Alternatively, ligation of membrane-associated ASF with the antibody may, by some mechanism, lead to activation of the APC, rendering it more potent at stimulating T-cell proliferation. If this first hypothesis is correct, then it is tempting to speculate that ASF may serve a regulatory or suppressive function in vivo. Likewise, if the alternative hypothesis is correct, then ASF may serve a role in augmenting T-cell-mediated processes. The third hypothesis is that the ASF receptor is expressed by T regulatory cells and that engagement of this receptor activates the suppressive functions of this population. In this instance, binding of TLD-1A8A to ASF would interfere with this interaction, resulting in no suppressive activities by the T-regulatory cell. In any event, the in vivo function of ASF could be significant, and warrants further investigation.

Recent evidence has suggested a beneficial effect of ASF in patients with Meniere's disease. ${ }^{4,29}$ The suggested mechanism for the beneficial effect of ASF in this disease is a decrease in the production of endolymph. However, an inflammatory pathogenesis of Meniere's disease has also been postulated..$^{30,31}$ The potential of ASF to serve as an anti-inflammatory agent should also be considered as a potential mechanism.

At this time, we can conclude that ASF is a molecule about which more needs to be known. Its ability to counteract highly potent enterotoxins is dramatic. Likewise, the apparent immunoregulatory role it plays in T-cell-mediated reactions could be very important. It is too soon to determine whether ASF exerts its effects via the same cellular or subcellular mechanisms, utilizing the same receptors, in the intestine as in immune reactions. Nevertheless, in the investigation of the biology and physiology of ASF, its participation in numerous immunological reactions must now be sought.

\section{Acknowledgement}

Flow cytometry and confocal microscopy was performed at Dartmouth Medical School in The Herbert C Englert Cell Analysis Laboratory, which was established by equipment grants from the Fannie E Rippel Foundation, the NIH Shared Instrument Program and Dartmouth Medical School and is supported in part by the Core Grant (CA 23108) from the National Cancer Institute to the Norris Cotton Cancer Center.

The authors would like to thank James D Gorham, (MD, PhD) for helpful discussions and a critical reading of this manuscript, Dr Srinivasa Nagalla for preparation of the microglial cDNA library and Brenda Barry for invaluable assistance in library screening.

This work was supported by NIH Grant NS047360-01 (WFH).

\section{References}

1 de Waal Malefyt R, Abrams J, Bennett B, et al. Interleukin 10 (IL-10) inhibits cytokine synthesis by human monocytes: an autoregulatory role of IL-10 produced by monocytes. J Exp Med 1991;174: 1209-1220.

2 Crisi GM, Santambrogio L, Hochwald GM, et al. Staphylococcal enterotoxin B and tumor-necrosis factor-alpha-induced relapses of experimental allergic encephalomyelitis: protection by transforming growth factor-beta and interleukin-10. Eur J Immunol 1995;25:3035-3040.

3 Kennedy MK, Torrance DS, Picha KS, et al. Analysis of cytokine mRNA expression in the central nervous system of mice with experimental autoimmune encephalomyelitis reveals that IL-10 mRNA expression correlates with recovery. J Immunol 1992;149:24962505.

4 Lange S, Lönnroth I. The antisecretory factor: synthesis, anatomical and cellular distribution, and biological action in experimental and clinical studies. Int Rev Cytol 2001;210:39-75.

5 Lönnroth I, Lange S. Purification and characterization of the antisecretory factor: a protein in the central nervous system and in the gut which inhibits intestinal hypersecretion induced by cholera toxin. Biochim Biophys Acta 1986;883:138-144.

6 Perry V. Microglia. In: Keane RH, (ed). Immunology of the Nervous System. Oxford University Press: New York, 1997.

7 Hickey WF. Basic principles of immunological surveillance of the normal central nervous system. Glia 2001;36:118-124.

8 Hickey WF. Leukocyte traffic in the central nervous system: the participants and their roles. Semin Immunol 1999;11:125-137.

9 Flaris NA, Densmore TL, Molleston MC, et al. Characterization of microglia and macrophages in the central nervous system of rats: definition of the differential expression of molecules using standard and novel monoclonal antibodies in normal CNS and in four models of parenchymal reaction. Glia 1993;7:34-40.

10 Williams KC, Zhao RW, Ueno K, et al. PECAM-1 (CD31) expression in the central nervous system and its role in experimental allergic encephalomyelitis in the rat. J Neurosci Res 1996;45:747-757.

11 Williams KC, Zhao W, Politopoulou G, et al. Inhibition of experimental allergic encephalomyelitis with an antibody that recognizes a novel antigen expressed on lymphocytes, endothelial cells, and microglia. Lab Invest 2000;80:313-326.

12 Male D, Rahman J, Linke A, et al. An interferoninducible molecule on brain endothelium which controls lymphocyte adhesion mediated by integrins. Immunology 1995;84:453-460.

13 Giulian D, Baker TJ, Shih LC, et al. Interleukin 1 of the central nervous system is produced by ameboid microglia. J Exp Med 1986;164:594-604.

14 Altschul SF, Madden TL, Schaffer AA, et al. Gapped BLAST and PSI-BLAST: a new generation of protein database search programs. Nucleic Acids Res 1997;25:3389-3402.

15 Johansson E, Lange S, Lönnroth I. Identification of an active site in the antisecretory factor protein. Biochim Biophys Acta 1997;1362:177-182. 
16 Hickey WF, Cohen JA, Burns JB. A quantitative immunohistochemical comparison of actively versus adoptively induced experimental allergic encephalomyelitis in the Lewis rat. Cell Immunol 1987;109:272281.

17 Mannie MD, Paterson PY, U’Prichard DC, et al. Induction of experimental allergic encephalomyelitis in Lewis rats with purified synthetic peptides: delineation of antigenic determinants for encephalitogenicity, in vitro activation of cellular transfer, and proliferation of lymphocytes. Proc Natl Acad Sci USA 1985;82:5515-5519.

18 Chou CH, Fritz RB, Chou FC, et al. The immune response of Lewis rats to peptide 68-88 of guinea pig myelin basic protein. I. T cell determinants. J Immunol 1979;123:1540-1543.

19 Griffin AC, Zhao W, Wegmann KW, et al. Experimental autoimmune insulitis. Induction by $\mathrm{T}$ lymphocytes specific for a peptide of proinsulin. Am J Pathol 1995;147:845-857.

20 Johansson E, Jennische E, Lange S, et al. Antisecretory factor suppresses intestinal inflammation and hypersecretion. Gut 1997;41:642-645.

21 Robinson AP, White TM, Mason DW. Macrophage heterogeneity in the rat as delineated by two monoclonal antibodies MRC OX-41 and MRC OX-42, the latter recognizing complement receptor type 3 . Immunology 1986;57:239-247.

22 Helmke RJ, German VF, Mangos JA. A continuous alveolar macrophage cell line: comparisons with freshly derived alveolar macrophages. In Vitro Cell Dev Biol 1989;25:44-48.
23 Kawahara H, Kasahara M, Nishiyama A, et al. Developmentally regulated, alternative splicing of the Rpn10 gene generates multiple forms of $26 \mathrm{~S}$ proteasomes. Embo J 2000;19:4144-4153.

24 Glickman $\mathrm{MH}$, Rubin DM, Fu H, et al. Functional analysis of the proteasome regulatory particle. Mol Biol Rep 1999;26:21-28.

25 Sigrist CJ, Cerutti L, Hulo N, et al. PROSITE: a documented database using patterns and profiles as motif descriptors. Brief Bioinform 2002;3: $265-274$

26 Lönnroth I, Lange S. Intake of monosaccharides or amino acids induces pituitary gland synthesis of proteins regulating intestinal fluid transport. Biochim Biophys Acta 1987;925:117-123.

27 Lönnroth I, Lange S, Skadhauge E. The antisecretory factors: inducible proteins which modulate secretion in the small intestine. Comp Biochem Physiol A 1988;90:611-617.

28 Lange S, Martinsson K, Lönnroth I, et al. Plasma level of antisecretory factor (ASF) and its relation to postweaning diarrhoea in piglets. Zentralbl Veterinarmed B 1993;40:113-118.

29 Hanner P, Jenniche E, Lange S. Antisecretory factor: a clinical innovation in meniere's disease. Acta Otolaryngologica 2003;123:779-780.

30 Mancini F, Catalani M, Carru M, et al. History of Meniere's disease and its clinical presentation. Otolaryngol Clin North America 2002;35:565-580.

31 Tomiyama S. Immuno-pathogenesis in Meniere's disease. J Nippon Med Sch 2003;70:212-218. 